\title{
Effects of Propranolol on the QT Dispersion in Congestive Heart Failure
}

\author{
Evandro Tinoco Mesquita, Flávia Cristina Carvalho de Deus, Cassia Regina Guedes, Eduardo Reis \\ Maia, César Gerson Pereira Subieta, Humberto Villacorta, Patrice Alessandra dos Santos, Helena \\ Cramer, Valeria Battistella Amado dos Santos, Luis José Martins Romeo
}

Niterói, RJ - Brazil

\begin{abstract}
Objective - Studies have shown that therapy with beta-blockers reduces mortality in patients with heart failure. However, there are no studies describing the effects of propranolol on the QT dispersion in this population. The objective of this study was to assess the electrophysiological profile, mainly QT dispersion, of patients with heart failure regularly using propranolol.
\end{abstract}

Methods - Fifteen patients with heart failure and using propranolol were assessed over a period of 12 months. Twelve-lead electrocardiograms (ECG) were recorded prior to the onset of beta-blocker therapy and after 3 months of drug use.

Results - A significant reduction in heart rate, in $Q T$ dispersion and in QTc dispersion was observed, as was also an increase in the PR interval and in the QT interval, after the use of propranolol in an average dosage of $100 \mathrm{mg} /$ day.

Conclusion - Reduction in QT dispersion in patients with heart failure using propranolol may explain the reduction in the risk of sudden cardiac death with beta-blocker therapy, in this specific group of patients.

Key words: congestive heart failure, electrocardiography, beta-blocker

Hospital Universitário Antônio Pedro - Niterói

Mailing address: Evandro Tinoco Mesquita - Av. Comandante Ari Parreiras, 311/

1102 - 24230-320 - Niterói, RJ, Brazil.

Received on $1 / 26 / 99$

Accepted on $4 / 24 / 99$
Currently, heart failure (HF) of all the cardiovascular diseases is a major public health problem. In addition to the significant impact on quality of life, $\mathrm{HF}$ drastically reduces the patient's survival and accounts for a substantial percentage of deaths caused by cardiovascular diseases ${ }^{1}$.

Death in patients with HF may result from progressive failure of the heart pump, or in more than half of the patients it may be sudden, probably resulting from arrhythmias ${ }^{1}$.

A measurement that may be very useful in this context and that has been identified as a marker of electrical myocardial instability is the variation in the duration of the QT interval between the leads, called QT dispersion.

QT dispersion is a new death predictor of $\mathrm{HF}$, and it reflects variations in the repolarization in different regions of the myocardium. This results from reentrant mechanisms due to the existence of areas of slow conduction, which constitute important physiological substrate for the arrhythmias. It has already been demonstrated that heterogeneity of ventricular repolarization is associated with a higher vulnerability to arrhythmias and occurrence of sudden death ${ }^{2}$.

Beta-blockers have been described as capable of reducing cardiovascular mortality after episodes of acute myocardial infarction (AMI) ${ }^{3}$, particularly reducing the incidence of fatal arrhythmias. The behavior of the QT dispersion in patients with HF using beta-blockers, however, has not yet been elucidated. This study analyzes prospectively the variations of the QT dispersion in the surface electrocardiogram (ECG) of patients with HF, before and after the use of propranolol, to assess the electrophysiologic effect of the drug on an important marker of the risk of sudden death.

\section{Methods}

From January ' 97 to January '98, 27 patients with HF according to the Framingham criteria ${ }^{4}$ and with a reduced ejection fraction on echocardiography $(<25 \%$, according to Simpson's method) sought medical assistance at the Heart Failure Outpatient Care Unit of the Hospital Universitário 
Antônio Pedro. The exclusion criteria for the use of propranolol were the following: atrial fibrillation, sinus bradycardia lower than $60 \mathrm{bpm}$, PR interval $>0.25 \mathrm{~s}$, bronchial asthma, intermittent claudication, and decompensated diabetes mellitus. All patients signed a written consent to take part in the study, which was approved by the Committee on Ethics in Research of the Universidade Federal Fluminense.

After a clinical stabilization period of 3 months, during which the patients used digoxin $(0.25 \mathrm{mg} /$ day $)$, enalapril (mean dose of 20mg/day), and sometimes diuretics, 12-lead ECGs were recorded at a rate of $25 \mathrm{~mm} / \mathrm{s}$. Treatment with propranolol was then started at an initial dose of $30 \mathrm{mg} /$ day. This dose was gradually increased (weak progression of $30 \%$ to $50 \%$ of the dose, according to clinical response) until the maximum dose of $120 \mathrm{mg} /$ day or the heart rate of 60 bpm, or both were achieved. Patients were hemodynamically stable during the entire period of assessment, $80 \%$ of whom were in functional class I and $20 \%$ in functional class II. The patients did not use any other cardioactive drugs and were instructed to seek the outpatient care unit in case of malaise, fatigue, edema, paroxysmal nocturnal dyspnea, asthma rales, and symptoms of hypotension or bradycardia (dizziness, somnolence, syncope). New ECGs were recorded after a mean period of 3 months of using propranolol.

ECG tracings were blindly analyzed by 2 independent investigators, and the following mean values of the variables were calculated: heart rate (HR), PR interval, QT interval duration, corrected QT (QTc), QT dispersion, and QTc dispersion, in 4 successive complexes for each lead. The QT interval was measured starting from the onset of the QRS complex until the end of the T wave, which is the return of the $\mathrm{T}$ wave to the baseline. When the $\mathrm{U}$ wave was present, the QT interval ended at the middle point between the T and $\mathrm{U}$ waves, which was obtained at the intersection of a tangent line to the repolarization line with the isoelectric line. QTc was obtained using Bazett's formula ${ }^{5}(\mathrm{QTc}=\mathrm{QT} / \sqrt{\mathrm{RR}})$. QT dispersion, defined as the difference between maximum and minimum QT, was calculated based on the QT intervals obtained in the 12 leads. The same was done for the QTc dispersion, which was corrected using the RR interval.

The results obtained were statistically analyzed, and the mean values of each variable were compared in the preand post-beta-blocker period, using a paired $t$ test and a significant value of $\mathrm{p}<0.05$.

\section{Results}

The study group consisted initially of 27 patients consecutively referred to the Heart Failure Outpatient Care Unit. Twelve out of these 27 patients were excluded from the study due to the following reasons: 2 had thyroid dysfunction (hypo- and hyperthyroidism), 4 had atrial fibrillation on the ECG, and 6 did not return to the outpatient care unit.

The 15 remaining patients underwent the protocol and were followed up from January '97 to January '98. This group consisted of 8 men $(53.3 \%)$, with a mean age of 51.9 years (age ranged from 28 to 72 years); $73.3 \%$ were white and $26.6 \%$ were black.
The etiology of the HF was ischemic or hypertensive, or both in 8 patients $(53.3 \%)$, idiopathic in $4(26.7 \%)$, and rheumatic, chagasic or alcoholic in the 3 remaining patients $(20.0 \%)$. The mean dosage of propranolol was $100 \mathrm{mg} /$ day, and the mean time of treatment was 3 months.

A clear change on the electrocardiographic profile of the patients with HF before and after the use of propranolol was observed.

Propranolol significantly reduced the HR and promoted a slight increase in the PR interval. An increase also occurred in the duration of the QRS complex and also in the duration of the QT interval. The maximum QT interval was obtained in one of the leads of the horizontal plane (V1 to V6) in $61 \%$ of the ECGs (out of the 26ECGs analyzed). There was no significant variation in the QTc; extension of the QT interval, therefore, may have resulted only from the concomitant reduction of the HR, because the QTc was not altered (table I).

The statistically significant reduction in the QT dispersion was the main finding, reflecting an electrophysiological improvement in these patients with HF caused by the use of propranolol.

QTc dispersion calculated in 13 patients also showed a significant reduction, indicating that the variation in the QT dispersion does not depend on the HR (fig. 1) ${ }^{6}$.

Bundle-branch blocks defined as QRS $>120 \mathrm{~ms}$ were documented in $4(16.0 \%)$ out of the 26 ECGs of the patients with HF, who could be analyzed. Left bundle-branch block (LBBB) was present in 3 patients (12.0\%) and right bundlebranch block $(\mathrm{RBBB})$ in only one patient $(4.0 \%)$. No relation between the values of the QT and QTc dispersions and the presence of LBBB and RBBB was observed.

\begin{tabular}{|lccc|}
\hline \multicolumn{4}{|c|}{ Table I - Electrocardiographic variations with the use of beta-blocker } \\
\hline ECG Variables & Pre-propranolol & Post-propranolol & p-value \\
\hline Heart rate & $83 \pm 17 \mathrm{bpm}$ & $68 \pm 13 \mathrm{bpm}$ & 0.01 \\
PR interval & $167 \pm 43 \mathrm{~ms}$ & $177 \pm 41 \mathrm{~ms}$ & 0.08 \\
QRS duration & $88 \pm 31 \mathrm{~ms}$ & $93 \pm 44 \mathrm{~ms}$ & 0.54 \\
QT interval & $347 \pm 52 \mathrm{~ms}$ & $390 \pm 57 \mathrm{~ms}$ & 0.01 \\
QTc & $402 \pm 32 \mathrm{~ms}$ & $409 \pm 40 \mathrm{~ms}$ & 0.48 \\
QT dispersion & $97 \pm 45 \mathrm{~ms}$ & $63 \pm 20 \mathrm{~ms}$ & 0.03 \\
QTc dispersion & $98 \pm 39 \mathrm{~ms}$ & $63 \pm 24 \mathrm{~ms}$ & 0.01 \\
\hline
\end{tabular}

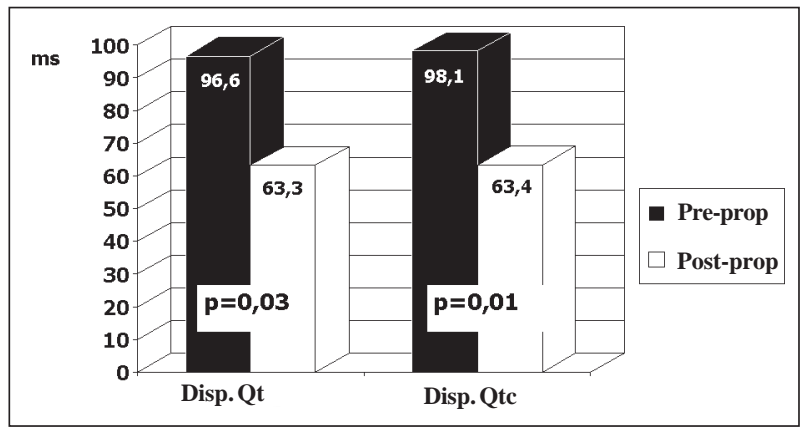

Fig. 1 - Variation of QT and QTc dispersions after the use of propranolol (Prop). 


\section{Discussion}

This study is the first to analyze the effects of propranolol on QT dispersion on the 12-lead ECG of patients with HF.

QT dispersion, QTc dispersion and the respective adjusted dispersions were observed to be significantly increased in patients with HF in this study, when compared with the normal values reported in the literature.

After 3 months of propranolol use at a mean dose of $100 \mathrm{mg} / \mathrm{day}$, an important reduction occurred in these values (fig. 2), suggesting a beneficial effect of the drug on myocardial electrophysiology in HF.

A number of studies have identified the QT dispersion as a marker of risk for sudden death ${ }^{7}$. Analysis of QT dispersion has been satisfactorily used to stratify the risks and to measure the effects of certain interventions on the ventricular repolarization. Baar et al ${ }^{1}$ showed that patients with HF, who suddenly evolved to death, had values of QT dispersion significantly higher than the values found in patients who died due to progressive HF or who survived. Buja et al ${ }^{8}$ reported that QT dispersion is greatly increased in patients with diastolic HF compared with healthy patients. They also showed that QT dispersion is increased in patients with severe ventricular arrhythmias or those who evolved to sudden death.

Day et al ${ }^{9}$ concluded that the variability of the QT interval on the surface ECG reflects the regional myocardial electrical activity, not representing just a technical artifact. The finding of reduction in QT dispersion in patients with different diseases treated with class III antiarrhythmic drugs- sotalol and amiodarone - supports this theory. Another study confirming the reliability of the QT dispersion as a marker of risk for sudden death was developed by Goldner et al ${ }^{10}$ and compares the QT dispersion with the high-resolution ECG. The study showed that QT dispersion is a sensitive indicator of spontaneous or induced ventricular tachyarrhythmias with a sensitivity of $88 \%$ and specificity of 57\%. Higham and Campbell ${ }^{11}$ observed high values of QT and QTc dispersion in patients in the first hours after AMI; reduction in these values occur with clinical evolution and use of thrombolytic agents.
In addition, among all risk factors analyzed by Pinsky et $\mathrm{al}^{12}$ in the study on sudden death in patients awaiting heart transplantation, QT dispersion appeared as the best predictor of death during the waiting period. In a huge contrast, in the measurements obtained on the 12-lead ECG, variability of the QT interval was a strong predictor of mortality. When the patients were divided into 2 groups (QT dispersion $<140 \mathrm{~ms}$ and QT dispersion $>140 \mathrm{~ms}$ ), differences of one year in mortality were observed (24\% and $56 \%$, respectively, $\mathrm{p}<0.05$ ). Analysis of QT dispersion allowed the identification of high-risk patients only through ECG, which is a simple, low-cost, noninvasive method, available in most centers.

Pinsky et al ${ }^{12}$ also reported that the extension of the QT interval did not prove to be a marker of risk for sudden death in patients with cardiac dysfunction. Extension of the QT interval seems not to reflect the alteration of myocardial ventricular repolarization. This alteration is indicated by QT dispersion.

Ventricular arrhythmias are common in HF, and they seem to have a multifactorial cause, including ventricular reentrant mechanisms. The reentrant circuit is formed by myocardial structural alterations, resulting in a slower and more heterogeneous ventricular repolarization ${ }^{13}$. As the QT interval reflects the duration of the myocardial action potentials, the increase in the QT interval variability, i. e., the increase in QT dispersion, acts as an indicator of susceptibility to possibly fatal ventricular arrhythmias ${ }^{14}$. The results of this study support the evidence that beta-blockers have a beneficial effect on HF, possibly due to a mechanism of reduction of the arrhythmogenic potential, which is expressed by a decrease in the QT dispersion on the ECG.

Previous studies have shown the effects of beta-blockers on the HF. Recent studies using carvedilol ${ }^{15}$, a nonselective beta-blocker with vasodilating properties, have shown results similar to ours, with significant reduction in the QT and QTc dispersions in patients with HF.

The mechanism remains unknown. It is suggested, however, that increased adrenergic tonus has a significant effect on QT dispersion ${ }^{16}$. Patients with the long QT syndrome

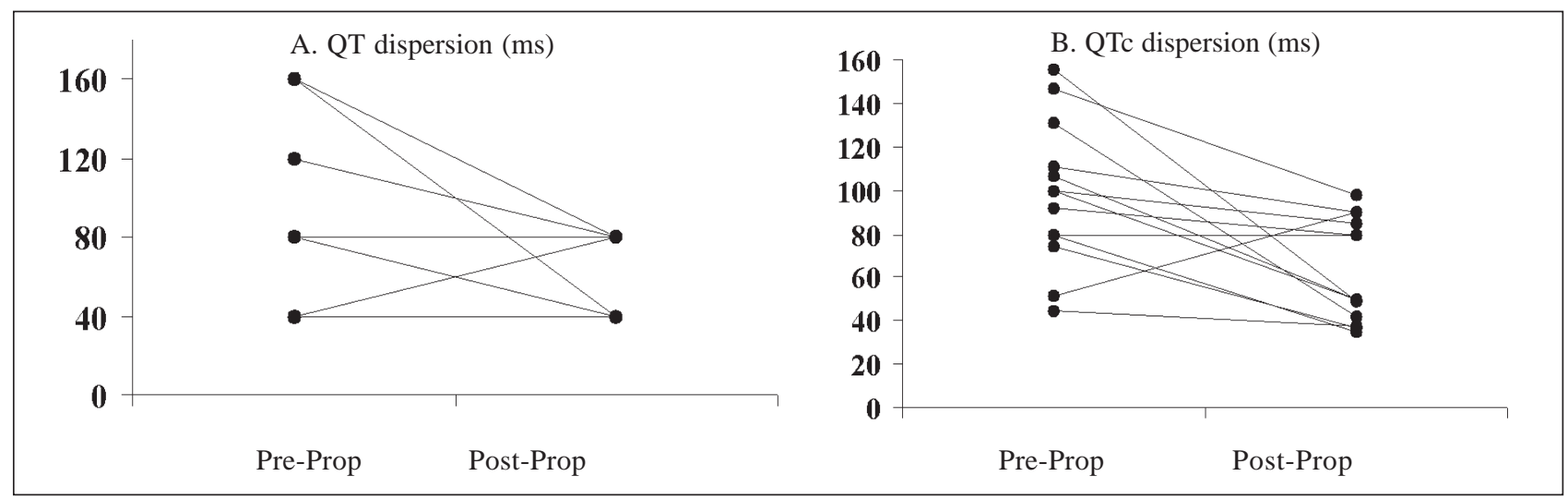

Fig. 2 - Individual variations of QT and QTc dispersions (A and B, respectively) with the use of propranolol (Prop). 
treated with beta-blockers or with sympathetic denervation showed reduction in QT dispersion. This finding is particularly appealing when the neurohumoral hypothesis of $\mathrm{HF}$, which suggests that the progression of the disease results from the chronic action of the adrenergic system ${ }^{16}$, is considered. Further investigations are still required; the excessive neuronal activation in HF may, however, influence the QT dispersion, promoting a physiopathological interaction with the heart mortality in these conditions.

Even though carvedilol is the paradigm beta-blocker in $\mathrm{HF}$, no comparative studies have been conducted comparing the less costly first-generation beta-blockers with carvedilol. In public hospitals, because of severe socioeconomic problems, patients with HF have difficulty paying for expensive treatments, especially those involving multiple drugs. This study has shown that the effect of propranolol on cardiac function is similar to that reported in the literature on the most recent drugs ${ }^{17,18}$.

This study has shown that a significant variation in the electrocardiographic tracings may be obtained after the use of propranolol. In addition, the ECGs were manually analyzed, and to minimize this bias, the tracings were separately assessed by two investigators, who ignored the clinical details of this complementary test.

Propranolol significantly reduced QT dispersion, which may constitute a possible marker of the reduction in the risk of sudden death. Further studies are still required, but this improvement in the electrophysiological profile of $\mathrm{HF}$ may be related to the reduction in sudden death observed with the use of beta-blockers.

\section{References}

1. Barr CS, Naas A, Freeman M, Lang CC, Struthers AD. QT Dispersion and sudden unexpected death in chronic heart failure. The Lancet 1994; 343: 327-9.

2. Merx W, Yoon MS, Han J. The role of local disparity in conduction and recovery time on ventricular vulnerability to fibrilation. Am Heart J 1977; 94: 603-10.

3. Chadda k, Goldstein S, Curb JD, Byington R. Effect of propranolol after acute myocardial infarction in patients with congestive heart failure. Circulation 1986; 73: 503-10.

4. Mc Kee PA, Castelli WP, Mc Namara PM, Kannel WB. The natural history of cardiac heart failure, The Framingham Study. NEngl J Med 1971; 285: 141.

5. BazettHC. An analysis of the time-relations of electrocardiograms. Heart 1920; 7:353.

6. MalikM,CammAJ. Mystery ofQTc Interval Dispersion. AmJCardiol 1997; 79:785-7.

7. Mantari M, Oikarinen L, Manninen V, Viitasalo M. QT dispersion as a risk factor for sudden cardiac death and fatal myocardial infarction in a coronary risk population. Heart 1997; 78: 268-72.

8. Buja G, Miorelli M, Turrini P, Melacini P, Nava A. Comparison of QT Dispersion in hypertrophic cardiomyopathy between patients with and without ventricular arrhythmias and sudden death. Am J Cardiol 1993; 72: 973-6.

9. Day CP, McComb JM, Campbell RWF. QT Dispersion: an indication of arrhythmia risk in patients with long QT intervals. Br Heart J 1990; 63: 342-4.

10. Goldner B, Brandspielgel HZ, Horwitz L, Jadonath R, Cohen TJ. Utility of QT Dispersion combined with the signal-averaged electrocardiogram in detecting patients susceptible to ventricular tachyarrhythmias. Am J Cardiol 1995; 76: 1192-3.
11. Higham PD, Campbell RWF. QT dispersion. Br Heart J 1994; 71: 508-10.

12. Pinsky DJ, Sciacca RR, Steinberg JS. QT Dispersion as a marker of risk in patients awaiting heart transplantation. J Am Coll Cardiol 1997; 29: 1576-84.

13. Perkiomaki JS, Koistinen MJ, Yli-Mayry S, Huikuri HV. Dispersion of QT interval in patients with and without susceptibility to ventricular tachyarrhythmias after previous myocardial infarction. J Am Coll Cardiol 1995; 26: 174-9.

14. Davey PP, Bateman J, Mulligan IP, Forfar C, Barlow C, Hart G. QT interval dispersion in chronic heart failure and left ventricular hypertrophy: relation to autonomic nervous and Holter tape abnormalities. Br Heart J 1994; 71: 268-73.

15. Gill AE Jr, Gilbert EM, Lowes BD, Abraham WT, Briston MR, Reiter MJ. Carvedilol Reduces QT Dispersion in Patients with Congestive Heart Failure. In: Anais da " $70^{\text {th }}$ Scientific Session Orange" (Supplement to Circulation). Orlando: Country Convention Center, 1997: I-577.

16. Fisher ML, Plotnick GD, Peters RW, Carliner NH. Beta-blockers in congestive cardiomyopathy - conceptual advance or contraindication? Am J Med 1986; 80(suppl 2B): 59-65.

17. Mesquita ET, Maia ER, Guedes CR, et al - Effects of propranolol on ventricular repolarization in patients with congestive heart failure. J Am Coll Cardiol 1998; 31(suppl C): 428C.

18. Maia ER, Subieta CGP, Tavares CMF, et al. Effects of propranolol on the parameters of systolic function in congestive heart failure. J Am Coll Cardiol 1998; 31 (suppl C): 428C. 